\title{
CEA-JSI Experimental Benchmark for validation of the modeling of neutron and gamma-ray detection instrumentation used in the JSI TRIGA reactor
}

\author{
Clément Fausser ${ }^{1}$, Nicolas Thiollay ${ }^{1}$, Christophe Destouches ${ }^{1}$, Loïc Barbot $^{1}$, Damien Fourmentel ${ }^{1}$, \\ Benoît Geslot ${ }^{1}$, Grégoire De Izarra ${ }^{1}$, Adrien Gruel ${ }^{1}$, Gilles Grégoire ${ }^{1}$, Christophe Domergue ${ }^{1}$, Vladimir \\ Radulović ${ }^{2}$, Tanja Goričanec ${ }^{2}$, Klemen Ambrožič², Gasper Žerovnik² ${ }^{2}$ Igor Lengar ${ }^{2}$, Andrej Trkov², \\ Žiga Štancar ${ }^{2}$, Anže Pungerčič² ${ }^{2}$ and Luka Snoj ${ }^{2}$ \\ ${ }^{1}$ CEA, DES, IRESNE, DER, SPESI, LDCI, Cadarache, F-13108 St-Paul-Lez-Durance, France \\ ${ }^{2}$ Reactor Physics Department, Jožef Stefan Institute, Jamova cesta 19, SI-1000 Ljubljana, Slovenia
}

clement.fausser@cea.fr

\begin{abstract}
Constant improvements of the computational power and methods as well as demands of accurate and reliable measurements for reactor operation and safety require a continuous upgrade of the instrumentation. In particular, nuclear sensors used in nuclear fission reactors (research or power reactors) or in nuclear fusion facilities are operated under intense mixed neutron and gamma-ray fields, and need to be calibrated and modeled to provide selective and accurate neutron and gamma-ray measurements.

The French Atomic Energy and Alternative Energies Commission (CEA) and the Jožef Stefan Institute (JSI) have started an experimental program dedicated to a detailed experimental benchmark with analysis using Monte Carlo particle transport calculations and a series of neutron and gamma-ray sensor types used in the JSI TRIGA Mark II reactor.
\end{abstract}

CEA has setup a simplified TRIPOLI-4® modeling scheme of the JSI TRIGA reactor based on the information available in the IRPhEP benchmark in order to facilitate analysis of future neutron and gamma-ray measurements. These allow the CEA to perform a TRIPOLI-4 instrumentation calculation scheme benchmarked with the JSI MCNP model.

This paper presents the main results of this CEA calculation scheme application and the analysis of their comparison to the JSI results obtained in 2012 with the MCNP5 \& ENDF/B-VII.0 calculation scheme. This paper will conclude with some information about the new experimental program to be carried out in 2022 in the TRIGA reactor core.

Keywords - Monte Carlo, TRIGA, TRIPOLI-4, MCNP, JEFF, IRDF, benchmark, fission chamber, fission rate.

\section{INTRODUCTION}

$\mathrm{T}$ HE collaboration on nuclear instrumentation between the CEA Experimental Physics, Safety experiment and
Instrumentation Section (SPESI) and the Reactor Physics Division of the Jožef Stefan Institute (JSI) started in 2008 in the frame of the bilateral agreement signed in 2006 between the CEA and the Slovenian Ministry of Higher Education, Science and Technology [1]. The mutual interest relies on the needs for a detailed neutron and gamma-ray characterization of the 250 $\mathrm{kW}$ TRIGA reactor for JSI and a need to diversify the available irradiation facilities for instrumentation testing for CEA.

The Instrumentation, Sensors and Dosimetry Laboratory (LDCI), a subsection of CEA SPESI, has been designing, manufacturing and using nuclear sensors dedicated to R\&D and power nuclear reactors for a long time.

The reference model of the reactor core using Monte Carlo $\mathrm{N}$-particle transport code (MCNP) [2] was published in a detailed NEA report available in the International Reactor Physics Evaluation Project (IRPhEP) handbook [3]. It revealed that the detailed MCNP5 model of the TRIGA reactor provides a satisfactory agreement with the measurements: The average deviations within the active fuel height are indeed less than 5\%, which is the approximate value of the combined uncertainty of measurements and modeling assumptions.

However, a set of experimental measurements could complete the characterization of the neutron flux and the photon flux of this benchmark. On the other hand, a comparison between the modeling schemes TRIPOLI-4 and MCNP should allow an improvement of the interpretation of the experimental results with in particular the recent improvement of the modelling of the prompt and delayed photons.

Thus, LDCI has proposed to develop a Monte Carlo model using the $4^{\text {th }}$ generation of the tridimensional Monte Carlo code for polykinetic continuous-energy radiation transport [4], TRIPOLI-4® version 11.0 (2020). This paper presents the TRIPOLI calculation scheme and the first analysis of the comparison of the results with those of the JSI one. 


\section{DESCRIPTION OF THE MEASURE: MINIATURE FISSION CHAMBERS}

The specificity of the measurements of the 2011-2012 benchmark lies on absolute fission rates measured by two miniature fission chambers (FC) provided by LDCI [5] (see Fig. 1), which assembled a ${ }^{235} \mathrm{U}$ fission chamber (FC5) and a ${ }^{238} \mathrm{U}$ fission chamber (FC8) in its fission chamber workshop at CEA Cadarache [6].
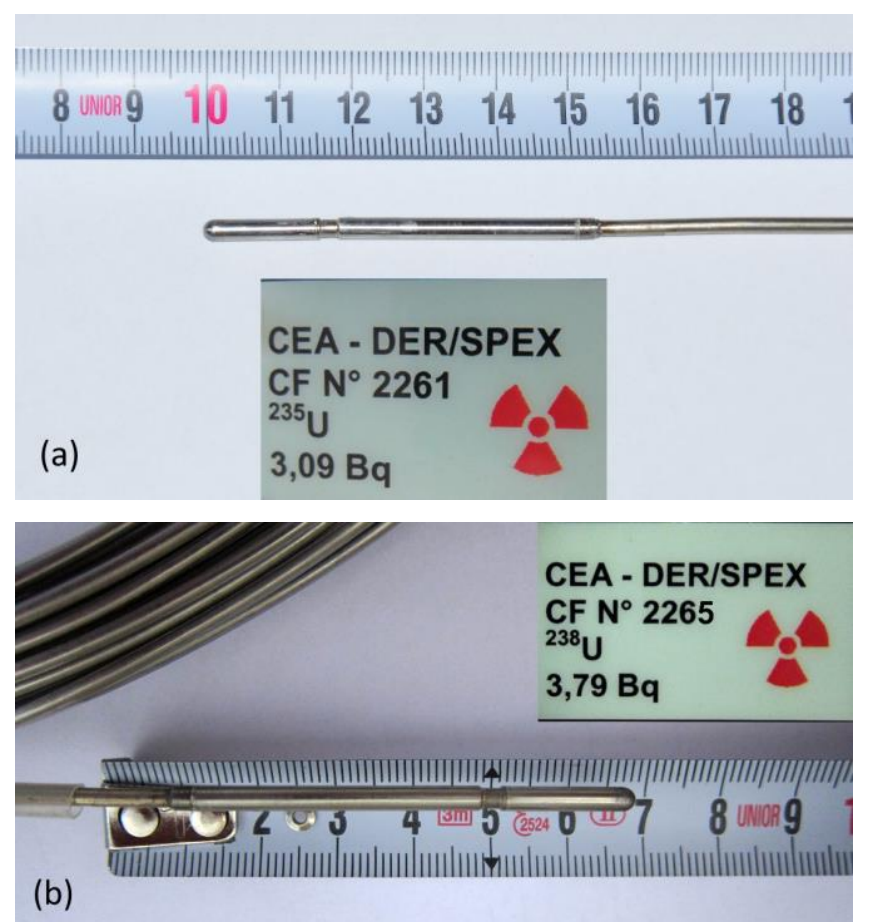

Fig. 1. ${ }^{235} \mathrm{U}$ miniature fission chamber "FC5" (a) and ${ }^{238} \mathrm{U}$ miniature fission chamber "FC8" (b).

Both FC5 and FC8 consist of a $4 \mathrm{~mm}$ long active part, which composition is detailed in NEA report [2]. The measurements were carried out in two different series of steady-state reactor power levels: one with FC5 at nominal power 103.5 W and the other with FC8 at $1034 \mathrm{~W}$. One should notice that the nominal power is determined based on measurement from ex-core neutron detector without taking into account the uncertainty and possible variations (e.g. due to control rods movements) of the conversion factor between the nominal factor and the FC.

Measurements were performed in the axial (Z) direction by a pneumatic system from above the core upper grid to below the core lower grid. The FCs are guided inside an aluminum tube filled with water. 9 measurement positions (MP) are explored as shown in the Fig. 2: 6 having a $8 \mathrm{~mm}$ diameter (MP14 to 15 and 21 to 23 ) and 3 around the central channel having a larger diameter (10.5 mm, MP17, 20 and 25).

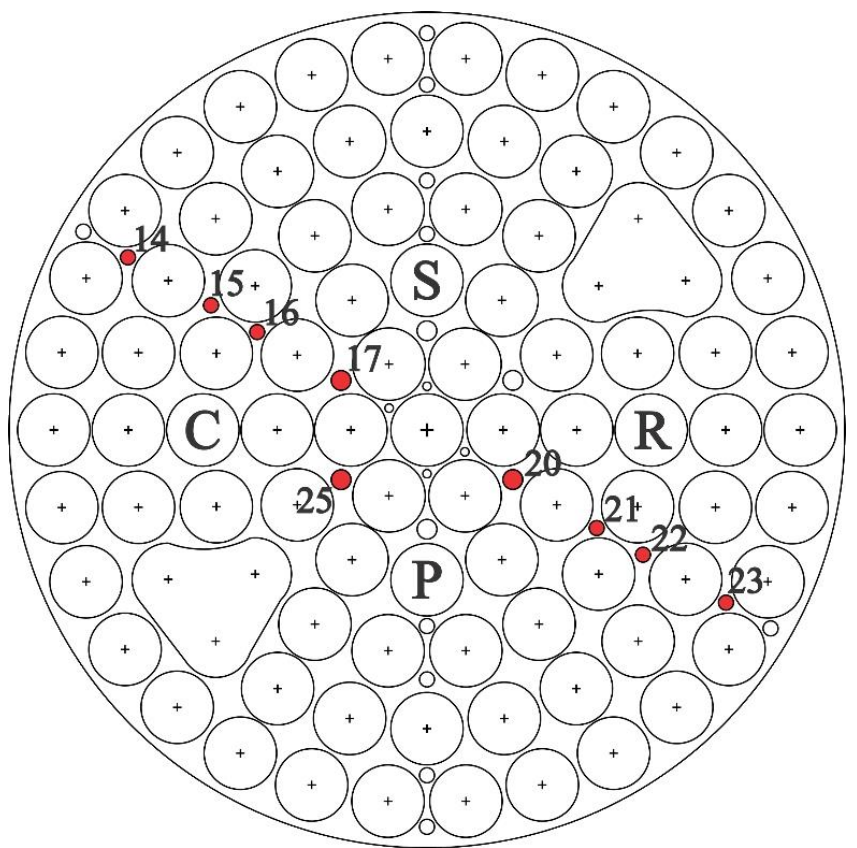

Fig. 2. Schematic of the JSI TRIGA core upper grid with denoted measuring positions.

\section{DESCRIPTION OF THE TRIPOLI-4 MODEL}

LDCI built the TRIPOLI-4 model applying four general principles.

The first principle is to be as rigorously identical as possible to the JSI MCNP model published in the NEA report. The MCNP model is translated in the TRIPOLI-4 format, keeping the same geometric and composition values, as well as identical modeling assumptions. For example, the fuel in standard fuel elements and fuel-follower control rods are modeled fresh, FCs are not physically depicted into their guide tubes (the fission rates being collected into a $4 \mathrm{~mm}$ long water cylinder instead), etc.

The second principle is to use surface-based volumes only (no TRIPOLI-4 combinatory volume nor ROOT-based volume [7], as a reminder "volume" in TRIPOLI-4 is the exact equivalent of a MCNP “cell”). Not only do these surface-based volumes speed-up computation, but in addition they can eventually supply CAD / MCNP / TRIPOLI-4 automatic converters or viewers, such as McCAD [8] or MCAM [9].

The third principle is to use the point-wise nuclear cross section library supplied by default with TRIPOLI-4.11.0, CEAv5.1.2. CEAv5.1.2 is mainly based on JEFF-3.1.1 [10] for transport cross-sections and contains point-wise IRDF-2002 cross-sections [11], which are used to calculate ${ }^{235} \mathrm{U}$ and ${ }^{238} \mathrm{U}$ fission rates in FCs.

The fourth principle is to use the same control rods positions as the two MCNP models used to provide the MCNP results in the NEA report.

The main difference between the TRIPOLI-4 and MCNP models lies in the absence of transformation matrixes in TRIPOLI-4.11.0. As the JSI TRIGA core is composed of 6 rings (with an additional one for the rotary groove) at regular angle pitch, 161 transformation matrixes allow the MCNP code to automatically copy and move each of the different generic geometries (one for a standard fuel elements, one for a fuelfollower control rod, etc.). For the TRIPOLI-4 model, LDCI 
created a LibreOffice Calc file for each generic geometry, which automatically generates TRIPOLI-4 surface-based volumes from the values of the MCNP transformation matrixes. The TRIPOLI-4 volumes obtained are then copied and pasted into the TRIPOLI-4 data file, which leads to a longer data file only for the geometry section.

Fig. 3 and Fig 4. respectively illustrate a side view and a top view of the TRIPOLI-4 model obtained in FC5 configuration.

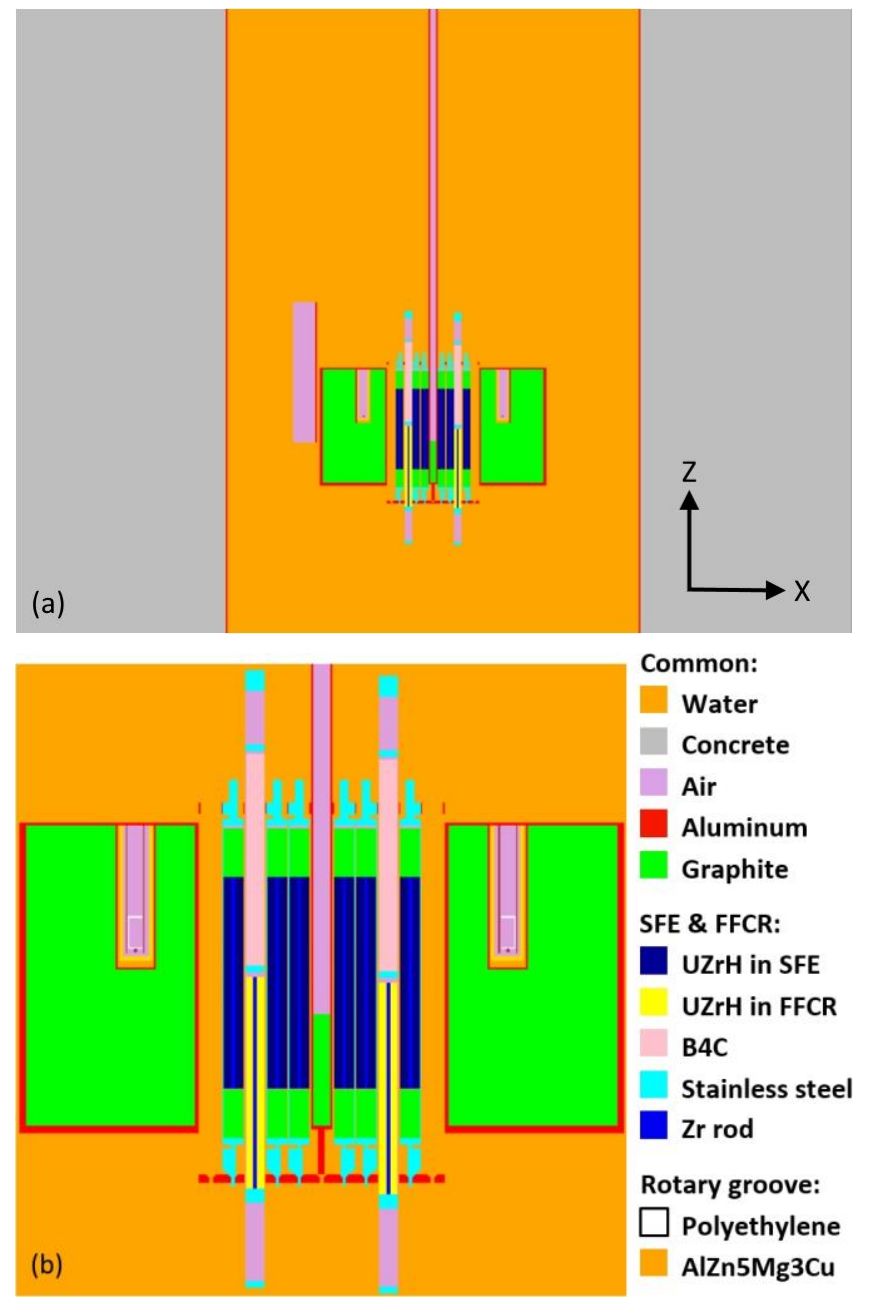

Fig. 3. $\mathrm{Y}=0 \mathrm{~cm}$ XZ side-view of FC5 TRIPOLI-4 JSI TRIGA model (a) with zoom on the core (b).

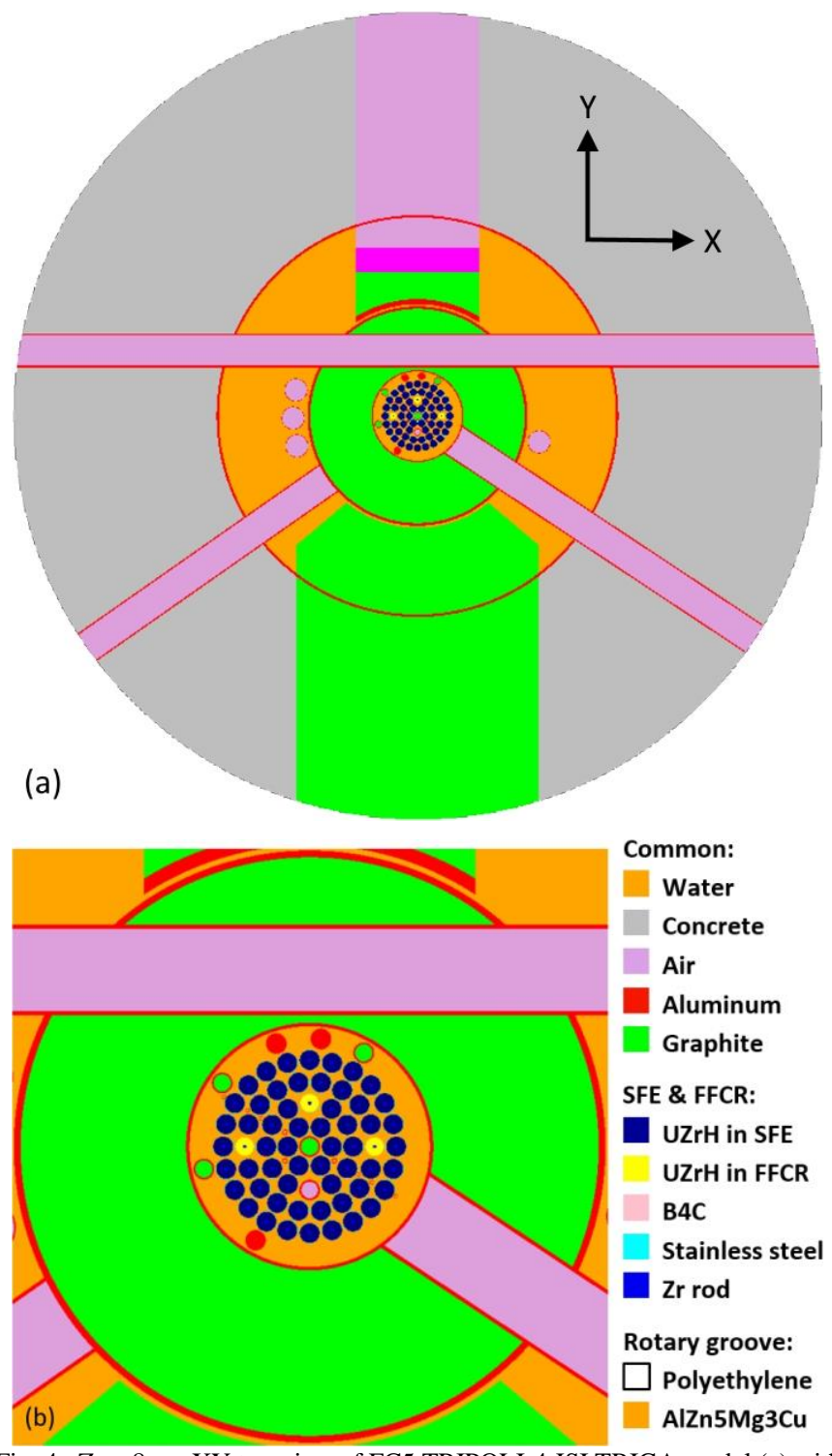

Fig. 4. $\mathrm{Z}=-8 \mathrm{~cm}$ XY top-view of FC5 TRIPOLI-4 JSI TRIGA model (a) with zoom on the core (b).

\section{FROM MONTE CARLO RAW RESUlTS TO ABSOLUTE TOTAL FISSION RATES}

As with the MCNP models, the neutron source is normalized to one in the TRIPOLI-4 models. Thus, two normalization factors (one for FC5 and the other for FC8) must be processed in order to convert the fission rates obtained in the MCNP or TRIPOLI-4 results in order to compare to the FC absolute fission rates. Even if the irradiations are always performed with a critical core ( $k_{\text {eff }}$ is exactly 1$)$, the use of fresh fuel in the models leads to $k_{\text {eff }}$ values different from 1 in the criticality calculations. The normalization factor $K$ to be applied to Monte Carlo microscopic fission rates to obtain the absolute macroscopic fission rates in FC5 or FC8 deposits is given in equation (1):

$$
K=\frac{P \cdot \bar{v}}{w_{f} \cdot k_{e f f}} \cdot N,
$$

With $P$ the thermal power (unit: W), $\bar{v}$ the average number of neutrons released per fission (no unit), $w_{f}$ the average deposited energy by fission event (unit: $\mathrm{J}$ ) and $N$ the number of uranium atoms in the FC deposit (unit: $\times 10^{+24}$ ). The unit of $K$ is then: 
$\times 10^{+24} / \mathrm{s}$. LDCI used for the present work the NEA report values for $P$ (103.5 W for FC5 and $1034 \mathrm{~W}$ for FC8), $\bar{v}(2.439)$ and $w_{f}$ (193.7 MeV converted to J).

LDCI calculates $N$ values for FC5 and FC8 of respectively $2.270 \times 10^{16}$ atoms and $2.319 \times 10^{17}$ atoms and obtained $k_{\text {eff }}$ values of 1.04829 for FC5 and 1.04857 for FC8 (with statistical uncertainties of $\pm 2.0 \mathrm{pcm}$ and $\pm 1.4 \mathrm{pcm}$ at $k_{\text {eff }}=1$ ) with TRIPOLI-4 models. The $k_{\text {eff }}$ values obtained with TRIPOLI4.11.0 \& CEAv5.1.2 (mainly based on JEFF-3.1.1) for FC5 and FC8 differ only by $-106 \mathrm{pcm}$ and $-108 \mathrm{pcm}$ from MCNP5 \& ENDF/B-VII.0 $k_{\text {eff }}$ results (i.e. $-0.10 \%$, MCNP statistical uncertainties being of $\pm 2 \mathrm{pcm}$ ). The recalculated values of the $K$ factors for FC5 and FC8 are respectively $1.76 \times 10^{+5}$ and $1.80 \times 10^{+7}$. These values differ by $-1.2 \%$ and $-1.4 \%$ from those published in the NEA report. In the present work, the $\mathrm{K}$ recalculated values are used not only for the TRIPOLI-4 results, but also for the MCNP ones (the MCNP results in the NEA report are multiplied by 0.9884 for FC5 and 0.9865 for FC8).

\section{ABSOLUTE FISSION RATES RESULTS: COMPARISON BETWEEN THE MODELING RESULTS AND THE BENCHMARK- MODEL MEASUREMENTS}

JSI carried out thorough uncertainty estimation work on raw absolute fission rates measurements to provide benchmarkmodel measurements, which take into account some biases due to the modeling assumptions, such as fresh fuel or absence of modeling of the real FC geometries. Only one small diameter MP and far from the central channel (MP14) and one large diameter MP and close to the central channel (MP25) are illustrated in the present work. These illustrations depict the comparisons between TRIPOLI-4.11.0 \& CEAv5.1.2 \& IRDF-2002 (TRI4), MCNP5 \& ENDF/B-VII.0 (MCNP), and benchmark-model measurements (Meas). Fig. 5 and Fig. 6 correspond respectively to FC5 and FC8 in MP14. Fig. 7 and Fig. 8 correspond respectively to FC5 and FC8 in MP25. A diagram of a standard fuel element at the same altitudes accompanies all figures to facilitate analysis. Similar to the corresponding figures in the NEA report for MCNP, a green line provides the results of a fine grid ( $4 \mathrm{~mm}$ window every $\mathrm{mm}$, as for MCNP) with dedicated dots with error bars when the altitude corresponds to an experimental position. As for MCNP, the error bars associated with TRI4 quadratically combine the Monte Carlo statistical uncertainty and $\pm 2.6 \%$ for FC5 or $\pm 2.7 \%$ for FC8 (corresponding to the combined uncertainties of the $K$ factor and the nuclear data). As published in the NEA report, the measurements error bars contain not only the uncertainty of the count rate of the real measurement, but also all the uncertainties of the modeling assumptions (e.g. fresh fuel). When TRI4 and benchmark-model measurements are compared in these figures, the present work uses the same convention as the NEA report for the error bars, i.e. a sum of the two uncertainties, $\sigma_{S U M}$, instead of a quadratic combination, $\sigma_{Q U A D}$. Nevertheless, in these figures, the comparison between TRI4 and MCNP uses $\pm \sigma_{Q U A D}$ error bars made by the quadratic combination of each of their statistical uncertainties and the nuclear data library uncertainties estimated in the NEA report as $\pm 1.4 \%$ for FC5 and $\pm 1.6 \%$ for FC8. $K$ factors being theoretically identical for TRI4 and MCNP, their combined uncertainty does not considers it.
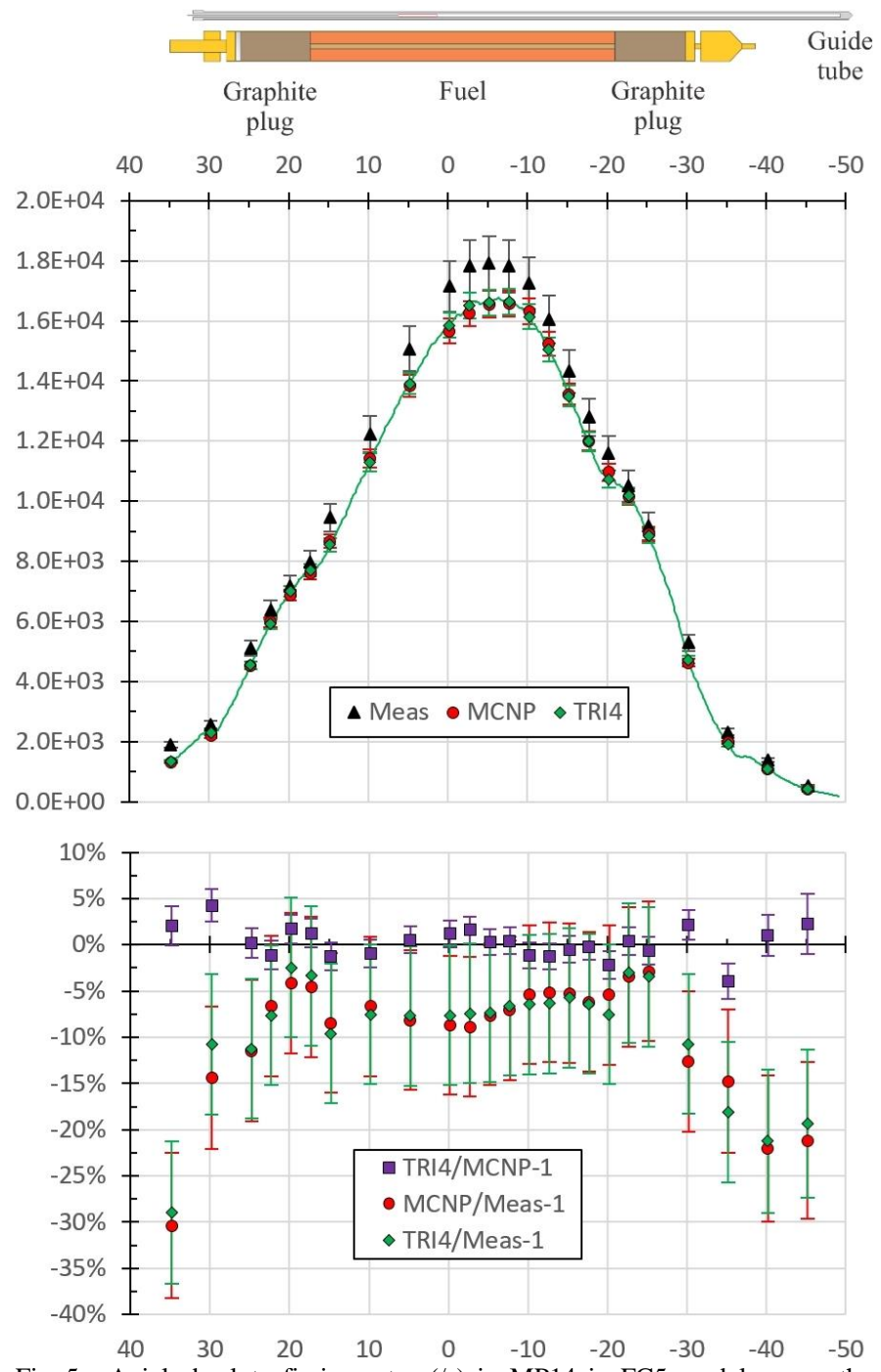

Fig. 5. Axial absolute fission rates (/s) in MP14 in FC5 model versus the altitude $\mathrm{Z}(\mathrm{cm})$ with a schematic of a standard fuel element at same altitudes.

As MCNP model absolute fission rates and benchmarkmodel measurements are accessible in the NEA report, only TRIPOLI-4 absolute fission rates are presented in the present work: FC5 ones in Table I and FC8 in Table II for every MPs, except MP20, which is in Table III for both FC5 and FC8. "Val." means the absolute fission rate and " $\sigma_{\text {Stat }}$ " means only the statistical uncertainty (i.e. without $\pm 2.6 \%$ for FC5 or $\pm 2.7 \%$ for FC8 due to the combined uncertainty of the $K$ factor and the nuclear data). 

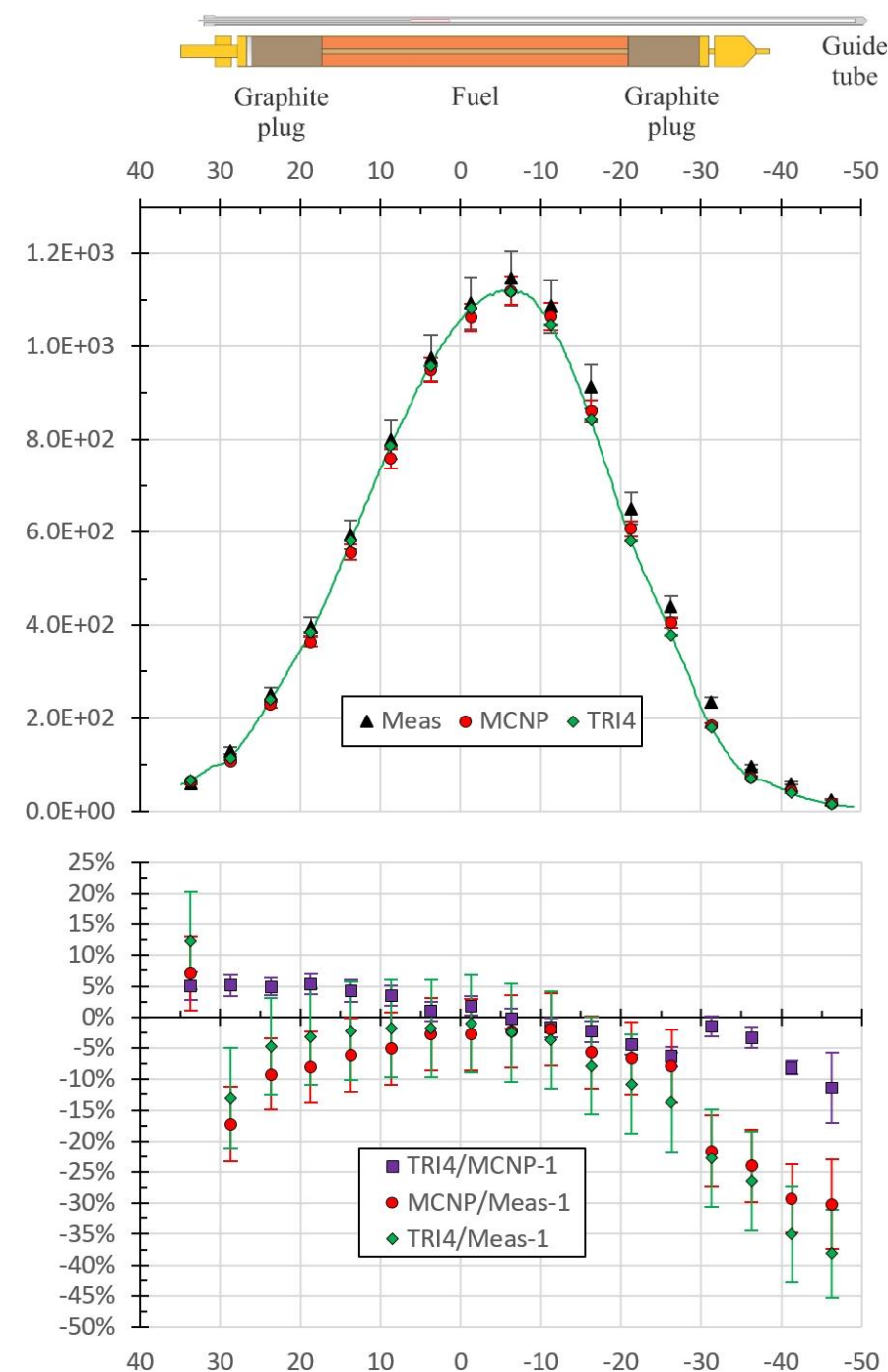

Fig. 6. Axial absolute fission rates (/s) in MP14 in FC8 model versus the altitude $\mathrm{Z}(\mathrm{cm})$ with a schematic of a standard fuel element at same altitudes.

Let consider the difference between TRI4 and MCNP relatively to their $\sigma_{Q U A D}:\left(\frac{T R I 4}{M C N P}-1\right) / \sigma_{Q U A D}$. Let now consider the axial profiles obtained in the 9 MPs, which correspond to a benchmark-model measurement set of 220 values for FC5 and 159 for FC8 (the $\mathrm{Z}=+35.7 \mathrm{~cm}$ altitude in MP20 is outside modeling volume for the MP, so it is not taken into account). $\left(\frac{T R I 4}{M C N P}-1\right) / \sigma_{Q U A D}$ is on average -0.7 for FC5 and -0.2 for FC8, with respective standard deviation of \pm 1.6 for FC5 and \pm 3.1 for FC8. Thus, there is a light negative bias between TRI4 and MCNP (especially for FC5) as well as standard deviations greater than one $\sigma_{Q U A D}$ (especially for FC8). However, the high standard deviation for FC8 masks a tendency to obtain positive values of $\left(\frac{T R I 4}{M C N P}-1\right)$ above $\mathrm{Z}=0$ and vice-versa below $\mathrm{Z}=0$, as in Fig. 6 and 8. At fuel altitudes in the standard fuel elements, $\left(\frac{\text { TRI4 }}{M C N P}-1\right)$ is on average only $-1.7 \%$ for FC5 and $+0.4 \%$ for FC 8 with standard deviation of $\pm 1.7 \%$ for FC5 and $\pm 3.0 \%$ for FC 8 . These values are lower than those of $\left(\frac{\text { MCNP }}{\text { Meas }}-1\right)$, which is on average $-4.7 \%$ for FC5 and $-3.6 \%$ for FC8 with standard deviation of $\pm 2.1 \%$ for FC5 and $\pm 3.3 \%$ for FC8. In addition, the standard deviations of $\left(\frac{T R I 4}{M C N P}-1\right)$ are also much lower than the uncertainties associated with the benchmark-model measurements, which are $\pm 5 \%$ for both FC5 and FC8. Therefore, TRI4 and MCNP give reasonably similar results with respect to the uncertainties of the benchmark-model measurements.

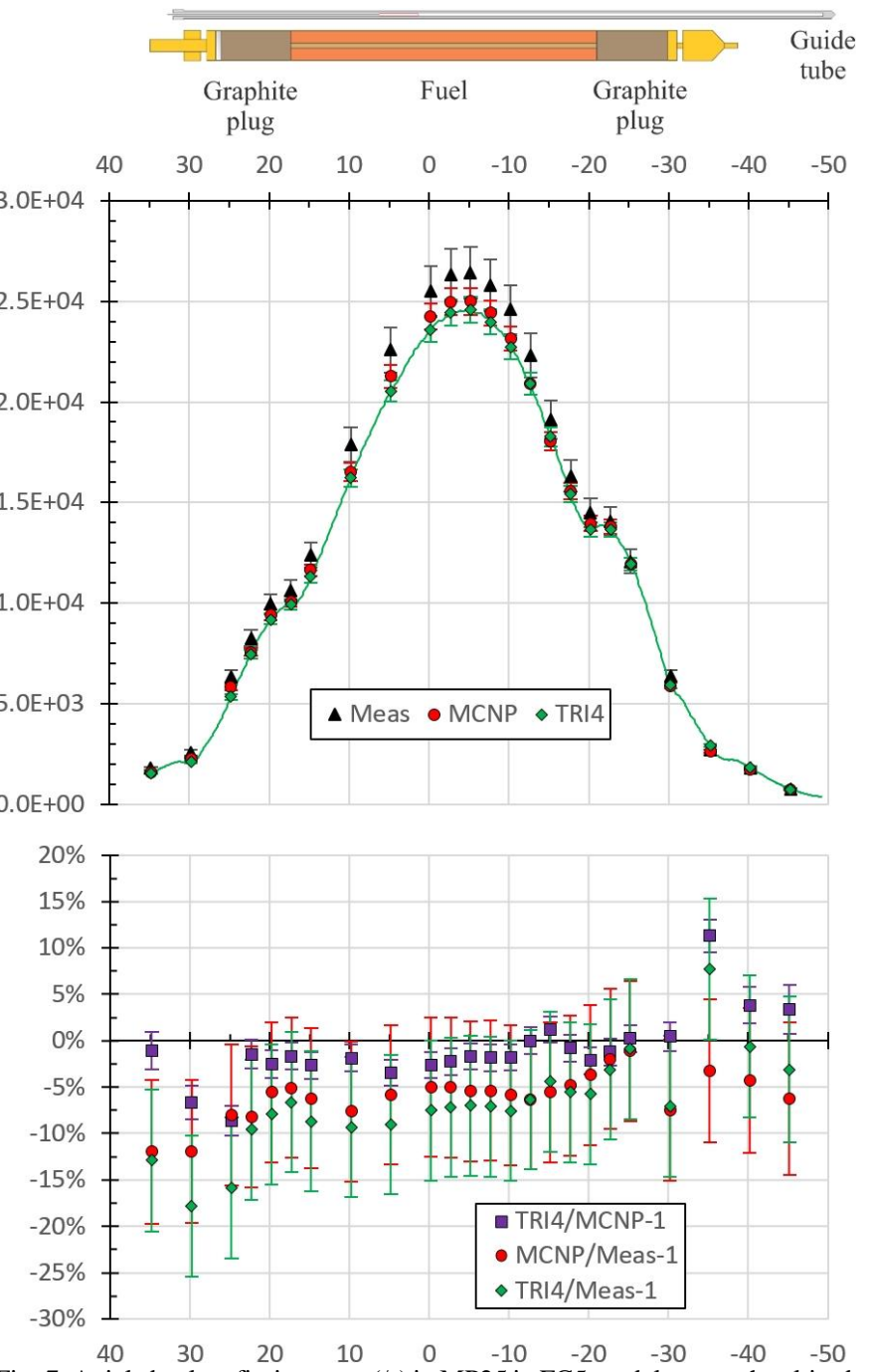

Fig. 7. Axial absolute fission rates (/s) in MP25 in FC5 model versus the altitude $\mathrm{Z}(\mathrm{cm})$ with a schematic of a standard fuel element at same altitudes.

Let now consider the difference between the absolute fission rates from TRI4 and from benchmark-model measurements with respect to their $\sigma_{Q U A D}$ in the 9 MPs at every altitude: $\left(\frac{T R I 4}{\text { Meas }}-1\right) / \sigma_{Q U A D}$. The average is -1.4 for FC5 and -1.8 for FC8, with respective standard deviations of \pm 1.0 for FC5 and \pm 1.8 for FC8. These values show a negative bias between TRI4 and the measurements and a standard deviation for FC8, which exceeds the expected \pm 1 value. Nevertheless, the study of $\left(\frac{T R I 4}{\text { Meas }}-1\right) / \sigma_{Q U A D}$ throughout the altitudes shows that, as encountered between MCNP and benchmark-model measurements, the Monte Carlo simulations are not satisfactory at extreme altitudes corresponding to the regions around the edge and outside of the active fuel height. 


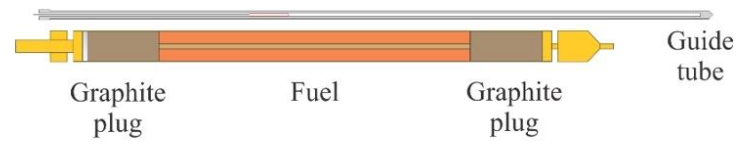

$\begin{array}{llllllllll}40 & 30 & 20 & 10 & 0 & -10 & -20 & -30 & -40 & -50\end{array}$
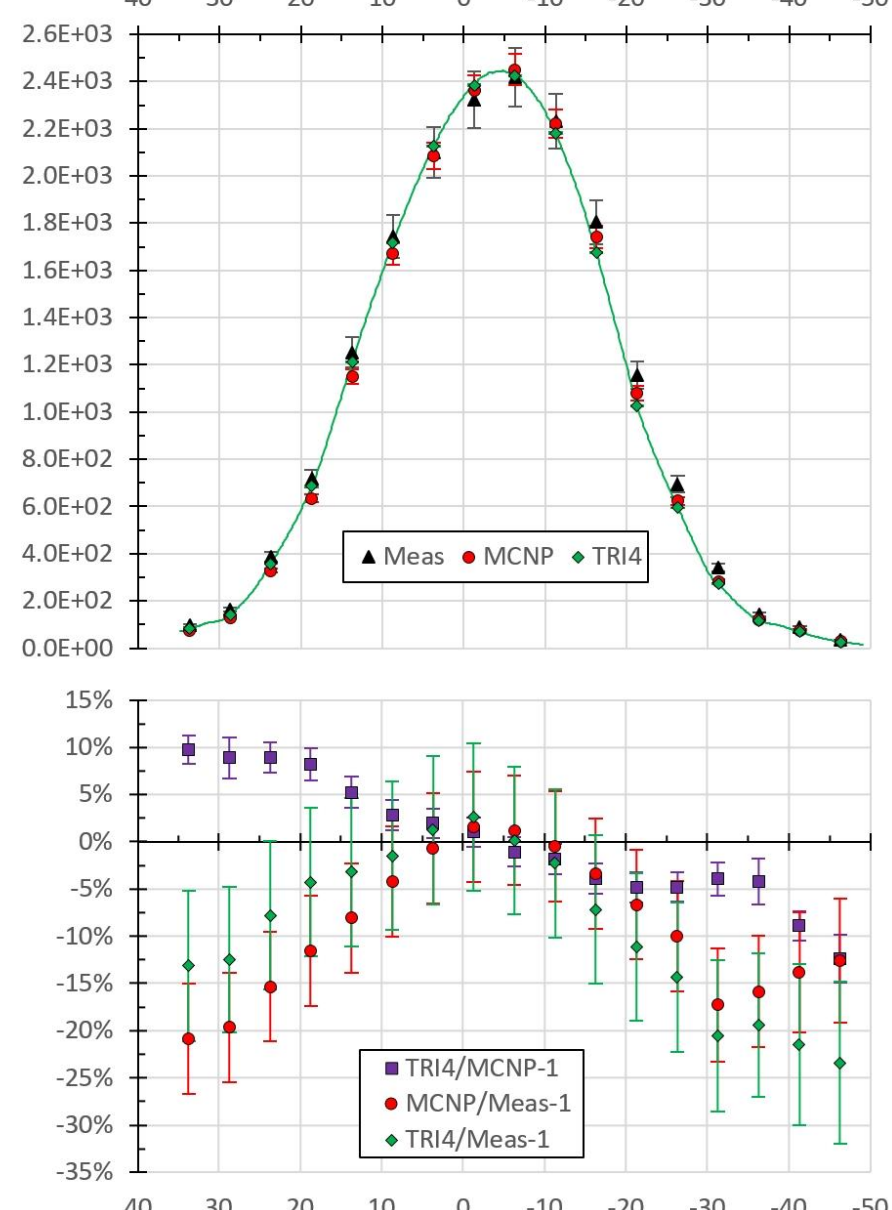

Fig. 8. Axial absolute fission rates (/s) in MP25 in FC8 model versus the altitude $\mathrm{Z}(\mathrm{cm})$ with a schematic of a standard fuel element at same altitudes.

However, $\left(\frac{T R I 4}{\text { Meas }}-1\right) / \sigma_{Q U A D}$ becomes much more satisfactory within fuel region of the standard fuel elements: The average is -1.1 for FC5 and -0.6 for FC8, with respective standard deviations of \pm 0.4 for FC5 and \pm 0.8 for FC8, with extreme values of $[-2.0,+0.5]$ for FC5 and $[-2.8,+0.8]$ for FC8. In terms of $\left(\frac{T R I 4}{\text { Meas }}-1\right)$, this corresponds to averages of $-6.3 \%$ for FC5 and $-3.2 \%$ for $\mathrm{FC} 8$, with respective standard deviations of $\pm 2.4 \%$ for FC5 and $\pm 4.4 \%$ for FC8, with extreme values of $[-11.1 \%,+3.0 \%]$ for FC5 and $[-16.2 \%,+4.7 \%]$ for FC 8 . The further away upwards or downwards from the altitude of the maximum absolute fission rates (close to $\mathrm{Z} \approx-4 \mathrm{~cm}$ ), the more the relative difference between the simulated absolute fission rates (either TRI4 or MCNP) and the benchmark-model measurements, relatively to the latter, increase. This phenomenon has already been noted in the NEA report. It may come from the fuel modeling assumptions, which consider only fresh fuel. The NEA report showed that this might have led to a large underestimation of the actual fission rates at the extreme parts of the fuel. However, this is not a major drawback if the TRI4 model is used for pre-design of the instrumentation. New experimental and modelling benchmark should allow solving this discrepancy. Nevertheless, for verification and validation purposes, both of the TRI4 and MCNP models using fresh fuel are not sufficiently satisfactory.

TABLE I

TRIPOLI-4.11.0 \& CEAv5.1.2 \& IRDF-2002 FC5 ABSOLUTE FISSION RATES (UNIT: PER SECOND)

\begin{tabular}{|c|c|c|c|c|c|c|c|c|}
\hline \multirow{2}{*}{$\begin{array}{c}\mathrm{Z} \\
(\mathrm{cm})\end{array}$} & \multicolumn{2}{|c|}{ MP14 } & \multicolumn{2}{|c|}{ MP15 } & \multicolumn{2}{|c|}{ MP16 } & \multicolumn{2}{|c|}{ MP17 } \\
\hline & Val. & $\sigma_{\text {Stat }}$ & Val. & $\sigma_{\text {Stat }}$ & Val. & $\sigma_{\text {Stat }}$ & Val. & $\sigma_{\text {Stat }}$ \\
\hline-45.2 & 427 & \pm 7 & 569 & \pm 8 & 655 & \pm 9 & 727 & \pm 9 \\
\hline-40.2 & 1081 & \pm 12 & 1311 & \pm 13 & 1482 & \pm 14 & 1792 & \pm 15 \\
\hline-35.2 & 1896 & \pm 15 & 2006 & \pm 15 & 2089 & \pm 16 & 2839 & \pm 18 \\
\hline-30.2 & 4716 & \pm 23 & 4295 & \pm 22 & 4505 & \pm 22 & 5894 & \pm 26 \\
\hline-25.2 & 8848 & \pm 32 & 9364 & \pm 32 & 9879 & \pm 33 & 11910 & \pm 36 \\
\hline-22.7 & 10188 & \pm 34 & 10447 & \pm 34 & 11114 & \pm 35 & 13598 & \pm 39 \\
\hline-20.2 & 10730 & \pm 35 & 9714 & \pm 32 & 10386 & \pm 33 & 13721 & \pm 38 \\
\hline-17.7 & 11980 & \pm 37 & 10484 & \pm 33 & 11279 & \pm 34 & 15618 & \pm 40 \\
\hline-15.2 & 13500 & \pm 39 & 12431 & \pm 36 & 13472 & \pm 37 & 18561 & \pm 44 \\
\hline-12.7 & 15041 & \pm 41 & 14071 & \pm 38 & 15312 & \pm 39 & 21233 & \pm 47 \\
\hline-10.2 & 16144 & \pm 42 & 15351 & \pm 40 & 16755 & \pm 41 & 23321 & \pm 49 \\
\hline-7.7 & 16645 & \pm 43 & 16185 & \pm 41 & 17616 & \pm 42 & 24525 & \pm 51 \\
\hline-5.2 & 16612 & \pm 43 & 16468 & \pm 42 & 18042 & \pm 43 & 25081 & \pm 51 \\
\hline-2.7 & 16508 & \pm 43 & 16153 & \pm 41 & 17792 & \pm 42 & 25012 & \pm 51 \\
\hline-0.2 & 15854 & \pm 42 & 15456 & \pm 40 & 16883 & \pm 41 & 24152 & \pm 50 \\
\hline+4.8 & 13919 & \pm 39 & 13034 & \pm 37 & 14298 & \pm 38 & 21096 & \pm 47 \\
\hline+9.8 & 11304 & \pm 36 & 10167 & \pm 33 & 11133 & \pm 34 & 16652 & \pm 41 \\
\hline+14.8 & 8553 & \pm 31 & 7046 & \pm 27 & 7618 & \pm 28 & 11457 & \pm 35 \\
\hline+17.3 & 7692 & \pm 29 & 6703 & \pm 27 & 7109 & \pm 27 & 10043 & \pm 32 \\
\hline+19.8 & 7000 & \pm 28 & 6681 & \pm 27 & 7067 & \pm 27 & 9230 & \pm 32 \\
\hline+22.3 & 5902 & \pm 26 & 5644 & \pm 25 & 5932 & \pm 25 & 7480 & \pm 29 \\
\hline+24.8 & 4541 & \pm 23 & 4085 & \pm 21 & 4251 & \pm 21 & 5378 & \pm 25 \\
\hline+29.8 & 2282 & \pm 15 & 1539 & \pm 12 & 1403 & \pm 12 & 2113 & \pm 15 \\
\hline+34.8 & 1347 & \pm 13 & 1076 & \pm 11 & 1154 & \pm 12 & 1548 & \pm 13 \\
\hline $\mathrm{Z}$ & \multicolumn{2}{|c|}{ MP21 } & \multicolumn{2}{|c|}{ MP22 } & \multicolumn{2}{|c|}{ MP23 } & \multicolumn{2}{|c|}{ MP25 } \\
\hline$(\mathrm{cm})$ & Val. & $\sigma_{\text {Stat }}$ & Val. & $\sigma_{\text {Stat }}$ & Val. & $\sigma_{\text {Stat }}$ & Val. & $\sigma_{\text {Stat }}$ \\
\hline-45.2 & 635 & \pm 9 & 538 & \pm 8 & 393 & \pm 7 & 738 & \pm 9 \\
\hline-40.2 & 1461 & \pm 13 & 1265 & \pm 12 & 984 & \pm 11 & 1799 & \pm 15 \\
\hline-35.2 & 2023 & \pm 15 & 1935 & \pm 15 & 1712 & \pm 14 & 2900 & \pm 18 \\
\hline-30.2 & 4400 & \pm 22 & 4136 & \pm 21 & 4259 & \pm 22 & 5918 & \pm 25 \\
\hline-25.2 & 9633 & \pm 32 & 8934 & \pm 32 & 8672 & \pm 32 & 11950 & \pm 36 \\
\hline-22.7 & 10798 & \pm 34 & 9972 & \pm 33 & 10059 & \pm 34 & 13632 & \pm 38 \\
\hline-20.2 & 9992 & \pm 32 & 9368 & \pm 32 & 10486 & \pm 35 & 13659 & \pm 38 \\
\hline-17.7 & 10835 & \pm 33 & 9889 & \pm 32 & 11569 & \pm 36 & 15420 & \pm 40 \\
\hline-15.2 & 12742 & \pm 36 & 11685 & \pm 35 & 13087 & \pm 39 & 18265 & \pm 43 \\
\hline-12.7 & 14628 & \pm 39 & 13202 & \pm 37 & 14375 & \pm 40 & 20901 & \pm 47 \\
\hline-10.2 & 15948 & \pm 40 & 14476 & \pm 39 & 15496 & \pm 42 & 22741 & \pm 49 \\
\hline-7.7 & 16781 & \pm 42 & 15174 & \pm 40 & 16287 & \pm 43 & 23972 & \pm 50 \\
\hline-5.2 & 17059 & \pm 42 & 15475 & \pm 40 & 16700 & \pm 44 & 24580 & \pm 51 \\
\hline-2.7 & 16761 & \pm 41 & 15195 & \pm 40 & 16888 & \pm 44 & 24424 & \pm 50 \\
\hline-0.2 & 15919 & \pm 40 & 14514 & \pm 39 & 16688 & \pm 43 & 23603 & \pm 50 \\
\hline+4.8 & 13622 & \pm 37 & 12373 & \pm 36 & 15136 & \pm 41 & 20543 & \pm 47 \\
\hline+9.8 & 10624 & \pm 33 & 9642 & \pm 32 & 12204 & \pm 37 & 16213 & \pm 41 \\
\hline+14.8 & 7310 & \pm 27 & 6732 & \pm 27 & 8774 & \pm 32 & 11318 & \pm 34 \\
\hline+17.3 & 6915 & \pm 27 & 6448 & \pm 26 & 7757 & \pm 30 & 9910 & \pm 32 \\
\hline+19.8 & 6864 & \pm 27 & 6418 & \pm 27 & 6981 & \pm 28 & 9169 & \pm 32 \\
\hline+22.3 & 5758 & \pm 25 & 5466 & \pm 25 & 5737 & \pm 26 & 7450 & \pm 29 \\
\hline+24.8 & 4107 & \pm 21 & 3893 & \pm 21 & 4214 & \pm 22 & 5327 & \pm 24 \\
\hline+29.8 & 1366 & \pm 11 & 1430 & \pm 12 & 1700 & \pm 13 & 2097 & \pm 15 \\
\hline+34.8 & 1092 & \pm 12 & 981 & \pm 11 & 895 & \pm 10 & 1540 & \pm 13 \\
\hline
\end{tabular}


TABLE II

TRIPOLI-4.11.0 \& CEAv5.1.2 \& IRDF-2002 FC8 ABSOLUTE FISSION RATES (UNIT: PER SECOND)

\begin{tabular}{|c|c|c|c|c|c|c|c|c|}
\hline \multirow{2}{*}{$\begin{array}{c}\mathrm{Z} \\
(\mathrm{cm})\end{array}$} & \multicolumn{2}{|c|}{ MP14 } & \multicolumn{2}{|c|}{ MP15 } & \multicolumn{2}{|c|}{ MP16 } & \multicolumn{2}{|c|}{ MP17 } \\
\hline & Val. & $\sigma_{\text {Stat }}$ & Val. & $\sigma_{\text {Stat }}$ & Val. & $\sigma_{\text {Stat }}$ & Val. & $\sigma_{\text {Stat }}$ \\
\hline-46.3 & 15 & \pm 0 & 20 & \pm 0 & 23 & \pm 0 & 26 & \pm 0 \\
\hline-41.3 & 39 & \pm 0 & 50 & \pm 0 & 56 & \pm 0 & 67 & \pm 0 \\
\hline-36.3 & 71 & \pm 0 & 80 & \pm 0 & 90 & \pm 0 & 112 & \pm 1 \\
\hline-31.3 & 181 & \pm 1 & 196 & \pm 1 & 215 & \pm 1 & 270 & \pm 1 \\
\hline-26.3 & 379 & \pm 1 & 452 & \pm 1 & 501 & \pm 1 & 592 & \pm 1 \\
\hline-21.3 & 581 & \pm 1 & 757 & \pm 1 & 857 & \pm 1 & 1028 & \pm 2 \\
\hline-16.3 & 841 & \pm 1 & 1187 & \pm 2 & 1399 & \pm 2 & 1707 & \pm 2 \\
\hline-11.3 & 1047 & \pm 2 & 1532 & \pm 2 & 1822 & \pm 2 & 2238 & \pm 2 \\
\hline-6.3 & 1118 & \pm 2 & 1692 & \pm 2 & 2019 & \pm 2 & 2492 & \pm 3 \\
\hline-1.3 & 1082 & \pm 2 & 1634 & \pm 2 & 1955 & \pm 2 & 2453 & \pm 3 \\
\hline+3.7 & 958 & \pm 2 & 1413 & \pm 2 & 1688 & \pm 2 & 2182 & \pm 2 \\
\hline+8.7 & 785 & \pm 1 & 1125 & \pm 2 & 1343 & \pm 2 & 1762 & \pm 2 \\
\hline+13.7 & 581 & \pm 1 & 788 & \pm 1 & 937 & \pm 2 & 1240 & \pm 2 \\
\hline+18.7 & 385 & \pm 1 & 471 & \pm 1 & 533 & \pm 1 & 693 & \pm 1 \\
\hline+23.7 & 240 & \pm 1 & 258 & \pm 1 & 285 & \pm 1 & 359 & \pm 1 \\
\hline+28.7 & 113 & \pm 1 & 100 & \pm 0 & 104 & \pm 1 & 141 & \pm 1 \\
\hline+33.7 & 66 & \pm 0 & 56 & \pm 0 & 62 & \pm 0 & 83 & \pm 0 \\
\hline $\mathrm{Z}$ & \multicolumn{2}{|c|}{ MP21 } & \multicolumn{2}{|c|}{ MP22 } & \multicolumn{2}{|c|}{ MP23 } & \multicolumn{2}{|c|}{ MP25 } \\
\hline$(\mathrm{cm})$ & Val. & $\sigma_{\text {Stat }}$ & Val. & $\sigma_{\text {Stat }}$ & Val. & $\sigma_{\text {Stat }}$ & Val. & $\sigma_{\text {Stat }}$ \\
\hline-46.3 & 22 & \pm 0 & 20 & \pm 0 & 14 & \pm 0 & 27 & \pm 0 \\
\hline-41.3 & 56 & \pm 0 & 49 & \pm 0 & 37 & \pm 0 & 68 & \pm 0 \\
\hline-36.3 & 90 & \pm 0 & 78 & \pm 0 & 65 & \pm 0 & 115 & \pm 1 \\
\hline-31.3 & 209 & \pm 1 & 189 & \pm 1 & 164 & \pm 1 & 271 & \pm 1 \\
\hline-26.3 & 488 & \pm 1 & 434 & \pm 1 & 359 & \pm 1 & 595 & \pm 1 \\
\hline-21.3 & 833 & \pm 1 & 725 & \pm 1 & 555 & \pm 1 & 1027 & \pm 2 \\
\hline-16.3 & 1340 & \pm 2 & 1124 & \pm 2 & 788 & \pm 1 & 1675 & \pm 2 \\
\hline-11.3 & 1737 & \pm 2 & 1447 & \pm 2 & 974 & \pm 2 & 2182 & \pm 2 \\
\hline-6.3 & 1919 & \pm 2 & 1593 & \pm 2 & 1074 & \pm 2 & 2423 & \pm 3 \\
\hline-1.3 & 1848 & \pm 2 & 1540 & \pm 2 & 1078 & \pm 2 & 2385 & \pm 3 \\
\hline+3.7 & 1604 & \pm 2 & 1341 & \pm 2 & 986 & \pm 2 & 2125 & \pm 2 \\
\hline+8.7 & 1286 & \pm 2 & 1076 & \pm 2 & 807 & \pm 1 & 1718 & \pm 2 \\
\hline+13.7 & 904 & \pm 2 & 760 & \pm 1 & 586 & \pm 1 & 1212 & \pm 2 \\
\hline+18.7 & 520 & \pm 1 & 452 & \pm 1 & 378 & \pm 1 & 686 & \pm 1 \\
\hline+23.7 & 279 & \pm 1 & 248 & \pm 1 & 226 & \pm 1 & 358 & \pm 1 \\
\hline+28.7 & 102 & \pm 0 & 94 & \pm 0 & 94 & \pm 0 & 142 & \pm 1 \\
\hline+33.7 & 59 & \pm 0 & 52 & \pm 0 & 48 & \pm 0 & 83 & \pm 0 \\
\hline
\end{tabular}

TABLE IIII

TRIPOLI-4.11.0 \& CEAv5.1.2 \& IRDF-2002 MP20 ABSOLUTE FISSION RATES

\begin{tabular}{crr} 
& \multicolumn{3}{c}{ (UNIT: } \\
\hline \hline $\mathrm{Z}$ & \multicolumn{2}{c}{ FC5 } \\
$(\mathrm{cm})$ & Val. & \multicolumn{1}{c}{$\sigma$ Stat } \\
\hline-45.2 & 750 & \pm 9 \\
-40.2 & 1779 & \pm 14 \\
-35.2 & 2854 & \pm 18 \\
-30.2 & 5907 & \pm 26 \\
-27.7 & 9132 & \pm 32 \\
-25.2 & 11900 & \pm 36 \\
-22.7 & 13483 & \pm 38 \\
-20.2 & 13588 & \pm 38 \\
-17.7 & 15216 & \pm 40 \\
-15.2 & 18086 & \pm 43 \\
-12.7 & 20599 & \pm 46 \\
-10.2 & 22435 & \pm 48 \\
-7.7 & 23660 & \pm 49 \\
-5.2 & 24302 & \pm 50 \\
-2.7 & 24132 & \pm 50 \\
-0.2 & 23297 & \pm 49 \\
+2.3 & 22095 & \pm 48 \\
+4.8 & 20415 & \pm 46 \\
+7.3 & 18336 & \pm 44 \\
+9.8 & 16066 & \pm 41 \\
+12.3 & 13612 & \pm 38 \\
+14.8 & 11157 & \pm 34 \\
+17.3 & 9883 & \pm 32 \\
+19.8 & 9127 & \pm 31 \\
+22.3 & 7444 & \pm 29 \\
+24.8 & 5291 & \pm 24 \\
+29.8 & 2042 & \pm 14 \\
+34.8 & 1535 & \pm 14 \\
\hline \hline
\end{tabular}

\section{CONCLUSION}

Thanks to the CEA-JSI benchmark published in the IRPhE Project handbook, LDCI was able to set up Monte Carlo model of the neutron transport in the JSI TRIGA Mark II reactor using the CEA reference code: TRIPOLI-4. Associated to a JEFF-3.1.1 library for transport cross-sections and IRDF-2002 for fission cross-sections. Results are satisfactory, compared to either the JSI MCNP5 \& ENDF/B-VII.0 model or the benchmark-model measurements results. Nevertheless, the use of fuel modeled fresh, without any absorbent fission product or transuranic elements, might be the reason why the simulations move away from the measurements outside the peak flux altitude and to a $k_{\text {eff }}$ value well above 1, whatever Monte Carlo code is used. Even if this is not a major drawback for instrumentation predesign, this simplification of fuel modeling may become problematic for validation and verification purposes, especially if coupled neutron and gamma-ray transport has to be considered. The new experimental campaigns scheduled in 2022, involving not only miniature fission chambers, but also thorough activation dosimetry campaign, some self-powered detectors, thermoluminescent dosimeters, ionization chambers and calorimeters, are expected to drastically improve the TRIPOLI-4 and MCNP models used by CEA and JSI. These new calculation schemes should use the most up-to-date Monte Carlo codes and nuclear data libraries, such as TRIPOLI-4.11.1 with JEFF-3.3 [12] and IRDFF-II [13] or MCNP6.2 [14] with ENDF/B-VIII.0 [15].

\section{ACKNOWLEDGMENT}

This work is carried out as part of a CEA-JSI 2019-2022 collaborative project within the CEA-MTZV bilateral 
agreement [1].

\section{REFERENCES}

[1] Christophe Destouches et al., "A Review of 10 years of JSI - CEA collaboration on nuclear instrumentation development," Proceedings of 27th International Conference Nuclear Energy for New Europe, NENE, 10-13 Sep. 2018, Portorož, Slovenia.

[2] X-5 Monte Carlo Team, "MCNP ${ }^{\mathrm{TM}}$ - A General Monte Carlo N-Particle Transport Code, Version 5," LA-UR-03-1987, 2004.

[3] Žiga Stancar, Luka Snoj, and Loïc Barbot, "Reaction Rate Distribution Experiments at the Slovenian JSI TRIGA Mark II Research Reactor," TRIGA-FUND-RESR-002, International Handbook of Evaluated Reactor Physics Benchmark Experiments. Paris: NEA/NSC/DOC(2006)1, OECD NEA, 2017.

[4] Emeric Brun et al., "TRIPOLI-4®, CEA, EDF and AREVA reference Monte Carlo code," Annals of Nuclear Energy, Vol. 82, 2015, pp. 151160, ISSN 0306-4549, doi: 10.1016/j.anucene.2014.07.053.

[5] Loïc Barbot et al., "Neutron Field Characterization of Irradiation Locations Applied to the Slovenian TRIGA reactor," 2013 3rd International Conference on Advancements in Nuclear Instrumentation, Measurement Methods and their Applications (ANIMMA), 2013, pp. 15, doi: 10.1109/ANIMMA.2013.6727954.

[6] Benoît Geslot et al., "Development and manufacturing of special fission chambers for in-core measurement requirements in nuclear reactors," 2009 1st International Conference on Advancements in Nuclear Instrumentation, Measurement Methods and their Applications, 2009, pp. 1-4, doi: 10.1109/ANIMMA.2009.5503816.

[7] René Brun and Fons Rademakers, "ROOT - An Object Oriented Data Analysis Framework, Proceedings AIHENP'96 Workshop," Lausanne, Sep. 1996, Nucl. Inst. \& Meth. in Phys. Res. A 389 (1997) 81-86.

[8] Lei Lu, Yuefeng Qiu, and Ulrich Fischer, "Improved solid decomposition algorithms for the CAD-to-MC conversion tool McCad," Fusion Eng. Des., 124 (2017), pp. 1269-1272.

[9] Lei Lu et al., "Application of MCAM in generating Monte Carlo model for ITER port limiter, Chinese Journal of Nuclear Science and Engineering," 27 (3) (2007), pp. 277-281.

[10] Arjan Koning et al., "Validation of the JEFF-3.1 Nuclear Data Library," Nuclear Energy Agency, OECD, 2013.

[11] "International Reactor Dosimetry File 2002," IAEA Technical Reports Series No. 452, STI/DOC/010/452 92-0-105106-9, 2006.

[12] Arjan J. M. Plompen et al. The joint evaluated fission and fusion nuclear data library, JEFF-3.3. Eur. Phys. J. A 56, 181 (2020), doi: 10.1140/epja/s10050-020-00141-9.

[13] Andrej Trkov et al., "IRDFF-II: A New Neutron Metrology Library," Nuclear Data Sheets, Vol. 163, 2020, pp. 1-108, ISSN 0090-3752, doi: 10.1016/j.nds.2019.12.001.

[14] Christopher J. Werner et al., "MCNP6.2 Release Notes", LA-UR-1820808, 2018.

[15] David A. Brown et al., "ENDF/B-VIII.0: The 8th Major Release of the Nuclear Reaction Data Library with CIELO-project Cross Sections, New Standards and Thermal Scattering Data," Nuclear Data Sheets, Vol. 148, 2018, pp 1-142, ISSN 0090-3752, doi: 10.1016/j.nds.2018.02.001. 